\title{
IR Optical Fiber-based Noncontact Pyrometer for Drop Tube Instrumentation
}

\author{
R.G. May, S. Moneyhun, W. Saleh, S. Sudcora, and R.O. Claus \\ Virginia Polytechnic Institute and State University, Fiber and Electro-Optics Research Center, \\ Blacksburg, VA 24061
}

A. M. Buoncristiani

NASA Langley Research Center, Hampton, 23665

\begin{abstract}
The design of a two-color pyrometer with an infrared optical fiber bundles for collection of the infrared radiation is described. The pyrometer design is engineered to facilitate its use for measurement of the temperatures of small, falling samples in a microgravity matcrials processing experiment using a 100-metcr long drop tube. Because the samples are small and move rapidly through the field-of-view of the pyrometer, the optical power budget of the detection system is severely limited. Strategies for overcoming this limitation are discussed.
\end{abstract}

\section{INTRODUCTION}

Optical fibers have becn applied to the measurement of temperature since $1980^{1}$. Methods may be broadly divided into intrinsic and cxtrinsic techniques. Intrinsic techniques include those in which light propagating in an optical fiber sensing element remains within the fiber; light in an extrinsic sensor instead interacts directly with the environment. Noncontact methods are inherently extrinsic because a physical separation between the fiber and the matcrial to be evaluated is required. Noncontact optical fiber-based temperature measurcment systems are typically two or three-wavelength pyrometers which utilize several optical detectors at several discretc wavelengths 2,3,4. The remote end of the optical fiber is aligned so that the hot material is within the fiber's ficld of view. Radiation which enters the fiber is split by an in-line coupler which independently feeds each of several optical filter/detector assemblics. As the temperature of the material to be sensed changes, the outputs of the different detectors also change, and via consideration of graybody radiation curves, the temperature of the material can be detcrmined.

An application of the described research is the determination of temperatures of falling particles in the Microgravity Matcrials Processing drop tube facility at the NASA Marshall Space Flight Center in Huntsville, AL. The drop tube is a 100 meter tall evacuated cylinder into which molten samples of metals or metallic binary alloys are dropped. During free-fall, the samples cool and solidify, yielding metallurgical structures that are not influenced by the carth's gravity. For a typical experiment, a sample is magnetically levitated at the top of the drop tube, and heated to melting by an RF induction furnace. The magnetic field is then turned off, and the sample is dropped. As the sample cools and solidifies during the drop, it undergoes recalescence due to the heat of fusion during the phase change. The temperature of the sample during the drop is calculated from heat transport equations, given the initial temperature (measured by an optical pyrometer adjacent to the RF induction furnace) and the clapse of time from release to recalcsence (determined by a silicon photodetector at the bottom of the drop tube). Experimental confirmation of the calculated temperature requires the use of noncontact measurement techniques that are independent of the usually unknown cmissivitics of the matcrials.

\section{MODELED PERFORMANCE OF FIBER OPTIC PYROMETER INPUT CONDITIONS}

The free fall of the heated pellet in the drop tube results in a limited time within the ficld-of-view of the fiber. Consecuently, the amount of radiant flux incident on the fiber face is very small. A mathematical model was derived to detcrmine the amount of radiant flux incident on the fiber face. Using this model a computer program was written 10 yictl numerical values representative of the proposed measurement system. 
The following assumptions were made:

(1) The pellet is falling through the geometric center of the drop-tube.

(2) The pellet is spherical in shape.

(3) The fiber face area is negligible compared to the pellet surface area.

(4) The radiation emitted by the pellet towards the fiber, as it is in the process of 'entering' and 'leaving' the field-of-view of the fiber, is neglected. In other words, the radiation when the pellet is in the field-of-view is considered to be constant from point (1) to point (2), as shown in figure (1).

(5) The radiation from the fiber towards the pellet is neglected.

(6) The velocity of the pellet within the field-of-view of the fiber is constant.

(7) The temperature of the pellet within the field-of-view of the fiber is constant.

One of the mathematical difficulties in treating radiative transfer between surfaces is accounting for the geometric relations involved in how the surfaces view each other. A method of accounting for the geometry is introduced in the form of a quantity called the "geometric configuration factor", greatly simplifying the analysis.

The configuration factor of the system is derived by considering the geometry as shown in Figure 1 . Figure 2 shows the fiber face area as a differential element $\mathrm{dA}_{1}$, and the pellet as a sphere of surface area $A_{2}$. The

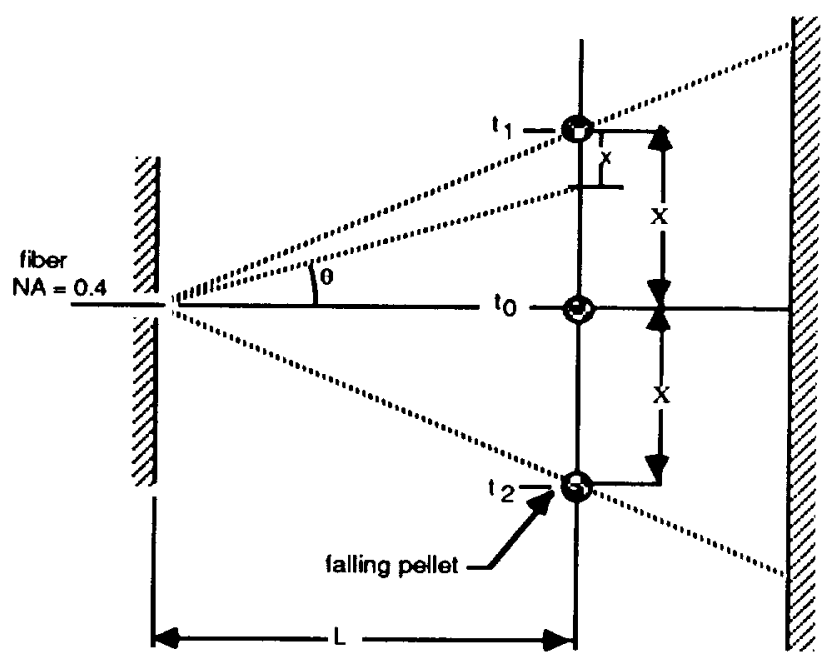

Figure 1. Geometry for radiative transfer model.

orientation is such that the normal to the center of the differential element passes through the center of the spherc. The standard result of the configuration factor of the system shown in Figure 2 is:

$$
F_{\mathrm{dt}-2}=\left[\frac{\mathrm{r}_{2}}{\mathrm{~h}}\right]^{2}
$$




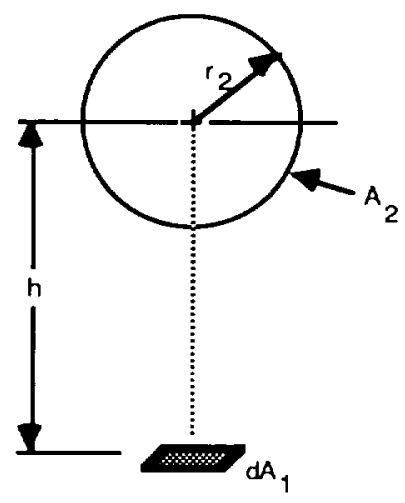

Figure 2. Geometry for configuration factor.

For the case when the pellet is directly in front of the fiber as shown in Figure 1, cquation (1) becomes:

$$
F_{\mathrm{d} 1-2}=\left[\frac{\mathrm{r}_{2}}{\mathrm{~L}}\right]^{2}
$$

where $\mathrm{L}=$ radius of the drop tube.

Since we are considering the flow of radiant flux from $\mathrm{A}_{2}$ to $\mathrm{dA}_{1}$, it is necessary to find $\mathrm{dF}_{2}-\mathrm{d} 1$ (the configuration factor needed for calculating energy flow from $A_{2}$ to $\mathrm{dA}_{1}$ ). The 'reciprocity relation' between a differential clement and a finite area states that

$$
A_{2} \mathrm{dF}_{2 \mathrm{dl}}=\mathrm{dAF} \mathrm{d} l-2_{2}
$$

or

$$
\mathrm{dF}_{2 \mathrm{dl}}=\frac{\mathrm{dA}_{1}}{\mathrm{~A}_{2}} \mathrm{~F}_{\mathrm{d} \mathrm{l}-2}
$$

where,

$$
\begin{aligned}
& \mathrm{dA}_{1}=\text { area of fiber face } \\
& \mathrm{A}_{2}=\text { surface area of the pellet. }
\end{aligned}
$$

Then equation (3) can be written as

$$
F_{2-d 1}=\frac{\pi_{1}^{2}}{4 \pi r_{2}^{2}}\left[\frac{r_{2}}{L}\right]^{2}
$$

The relation in equation (4) assumes that the normal to the fiber face passes through the center of the spherical pellet. Therefore, in order to consider for the cases when the pellet is at some angle, the component of the fiber face 
area is taken which is such that the normal to the component always passes through the center of the spherical pellet.

Therefore equation (4) becomes,

$$
F_{2-\mathrm{d} 1}=\frac{\left[\pi \pi_{1}^{2}\right] \cos \theta}{4 \pi \pi_{2}^{2}}\left[\frac{r_{2}}{L}\right]^{2}
$$

Now, $\theta$ is a function of " $x$ ", as shown in Figure (1):

$$
\cos \theta=\frac{\mathrm{L}}{\sqrt{\mathrm{L}^{2}+(\mathrm{X}-\mathrm{x})^{2}}}
$$

In equation (6), the quantities " $L "$ and " $X$ " are constant and " $\mathrm{x}$ " varies with time " $\mathrm{l}$ ".

Writing " $x$ " in terms of " $t$ ", we get:

$$
\mathrm{x}=\left(\frac{2 \mathrm{X}}{\Delta \mathrm{T}}\right) \mathrm{t}
$$

where,

$$
\begin{aligned}
& \Delta T=t_{1}-t_{0} \text { Time for which the pellet is in the ficld-of-vicw. } \\
& 2 X=\text { Total vertical distance traveled through the field-of-view. }
\end{aligned}
$$

and " $t$ " varies from $t_{1} t_{0} t_{2}$ as shown in Fig. (1).

Now, cquation (6) becomes,

$$
\cos \theta=\frac{L}{\sqrt{L^{2}+\left\{X-\left(\frac{2 X}{\Delta T}\right) t\right\}^{2}}}\left(\frac{r_{2}}{L}\right)^{2}
$$

Substituting (8) in (5), we get

$$
F_{2 d 1}=\frac{\left[\pi r_{1}^{2}\right.}{4 \pi r_{2}^{2}} \frac{L}{\sqrt{L^{2}+\left\{X-\left(\frac{2 X}{\Delta T}\right) t\right\}^{2}}}\left(\frac{r_{2}}{L}\right)^{2}
$$

Canceling the like terms from the numerator and denominator, we get: 


$$
F_{2-d 1}=\frac{r_{1}^{2}}{4 L} \frac{1}{\sqrt{L^{2}+\left\{X-\left(\frac{2 X}{\Delta T}\right) t\right\}^{2}}}
$$

To get the total incident radiant flux on the fiber face, we use the following equation:

$$
\mathrm{Q}_{2 \mathrm{~d} \mathrm{~d} l}=\sigma \mathrm{K}^{4} \mathrm{dF}_{2 \mathrm{dl}} \mathrm{A}_{2}
$$

where,

$$
\begin{aligned}
& \mathrm{dQ}_{2-\mathrm{d} 1}=\text { Flux incident on the fiber face. } \\
& \sigma=\text { Stephan }- \text { Boltzmann constant }=5.66961 \times 10^{-8} \mathrm{~W} / \mathrm{m}^{2} \mathrm{~K}^{4} \\
& \mathrm{~K}=\text { Temperature of pellet (source) } \\
& \mathrm{A}_{2}=\text { Surface area of the pellet. }
\end{aligned}
$$

As it is assumed that the temperature of the pellet remains constant during the short interval of time the pellet is in the ficld-of-view of the fiber, we find that equation (10) has all the terms constant except $F_{2-d 1}$, which varies with lime.

Equation (9) is integrated between " $t_{1}$ " and "to" and the result doubled to account for the time from "t0" $t_{0}$ " $t_{2}$ "

$$
\left[F_{2-d 1]}\right]_{1,0 t_{0}}=\int_{t_{1}}^{i_{0}} \frac{r_{1}^{2}}{4 L} \frac{1}{\sqrt{L^{2}+\left\{X-\left(\frac{2 X}{\Delta T}\right)\right\}^{2}}} d t
$$

Let

$$
A=L^{2}+X^{2}
$$




$$
\begin{aligned}
& B=-\left(\frac{4 X^{2}}{\Delta T}\right) \\
& C=\left(\frac{2 X}{\Delta T}\right)^{2}
\end{aligned}
$$

Substituting $A, B$ and $C$ in equation (11), we have

$$
\left[F_{2 d 1}\right]_{t_{1} t_{1} t_{0}}=\frac{r_{1}^{2}}{4 L} \int_{t,}^{l} \frac{1}{\sqrt{A+B t+C t}} d t
$$

Using the standard result for

$$
\int \frac{1}{\sqrt{x}} d x=\frac{1}{\sqrt{c}} \log \left[\sqrt{x}+x \sqrt{c}+\frac{b}{2 \sqrt{c}}\right]
$$

for $c>0$ and where $X=a+b x+c x^{2}$.

Then cquation (12) becomes

$$
\begin{aligned}
{\left[F_{2 d 1}\right]_{l_{1} \omega t_{0}}=\frac{r_{1}^{2}}{4 L} } & \left\{\frac{1}{\sqrt{C}} \log \left(\sqrt{A+B t_{0}+\mathrm{Ct}_{0}^{2}}\right)+t_{0} \sqrt{C}+\frac{B}{2 \sqrt{C}}\right\} \\
& \left.-\left\{\frac{1}{\sqrt{C}} \log \left(\sqrt{A+B t_{1}+\mathrm{Ct}_{1}^{2}}\right)+t_{1} \sqrt{C}+\frac{B}{2 \sqrt{C}}\right\}\right]
\end{aligned}
$$

Now,

$$
\left[\mathrm{F}_{2 \mathrm{~d} 1}\right]_{\text {total }}=2\left[\mathrm{~F}_{2 \mathrm{~d} 1}\right]_{1, \mathrm{bot}}
$$

Substituting equation (14) in equation (10), we get the total radiant flux incident on the fiber face.

\section{NUMERICAL SIMULATION}

The model for radiative transfer was coded into a FORTRAN computer program, yielding the resulting incident radiant flux in watts for different pellet temperatures. For these calculations the pellet was assumed to be $3 \mathrm{~mm}$ in diameter, and the single fiber was assumed to have a core diameter of $200 \mu \mathrm{m}$ and NA of 0.4 . Also, the drop tube radius was taken to be 6 inches.

The results show that the radiant flux incident on a $200 \mu \mathrm{m}$ fiber (with NA of 0.4), 10 meters down the drop-tube and at temperature $773 \mathrm{~K}$ is $0.45 \times 10^{-11}$ Watts. This number decreases further down the drop tube (e.g. at 190 
meters down the drop-tube it falls down to $0.5 \times 10^{-13}$ Watts). Further, at higher temperatures, we find that the amount of incident radiant flux increases. At a temperature of $3773 \mathrm{~K}$ and 10 meters down the drop-tube, the value becomes $0.25 \times 10^{-8}$ Watts. These numbers represent the ideal case as the falling pellet is assumed to be a blackbody. These numbers will further decrease if the falling pellet is considered to be a gray body and other effects (such as spectral attenuation of the fiber, inclusion of an anti-reflection coating, or stray reflections from the inside wall of drop-tube) are taken into account.

The field-of-view and hence the transit time of the pellet in the field-of-view can be increased by orienting the fiber at an angle with respect to the vertical wall of the drop tube. This approach is shown in Figure (3). Here $\beta$, the angle by which the fiber is tilted, is assumed to be less than $\theta$, where $\theta=\sin ^{-1}$ (NA), NA being the numerical aperture of the fiber. The configuration factor was adapted to account for the tilted fiber, and coded into a FORTRAN program. The results showed that the tilting of fiber increased the time in which the pellet remained in the field-of-view of the fiber, which, in tum, increased the radiant flux incident on the fiber-face. They indicate that the radiant flux incident on the fiber-face, 10 meters down the drop-tube, when it is tilted at an angle of $20^{\circ}$ with respect to the horizontal reference time is $0.87 \times 10^{-9}$ Watts which corresponds to a $17.6 \%$ increase in the incident radiant flux. Similarly, at 190 meters down the drop-tube, with the fiber tilted at $20^{\circ}$ with respect to the horizontal reference line it becomes $0.1 \times 10^{-10}$ Watts which corresponds to a $12.4 \%$ increase in the incident radiant tlux.

As shown by the results of the above programs that as we move down the drop-tube, both the time in which one pellet remains in the field-of-view and the incident radiant flux decrease appreciably. This implies that it will be necessary to increase the tilt angle $\beta$ as we move down the drop-tube, to compensate for the loss in the incident radiant flux.

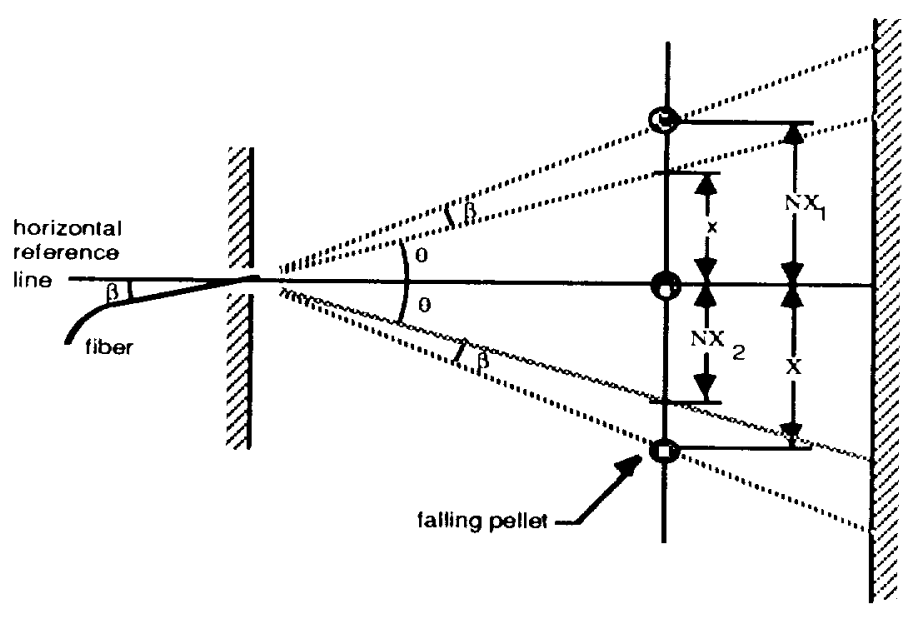

Figure 3. Geometry for tilted fiber.

\section{IMPLEMENTATION OF PYROMETER}

To augment the radiant flux incident on the detectors, an experimental pyrometer is being assembled with a six inch long, 0.125 inch diameter bundle of $200 \mu \mathrm{m}$ diameter core, $250 \mu \mathrm{m}$ diameter clad tirconium fluoride fibers. The bundle of approximately 120 fibers will increase the optical power budget at the detectors by about $20 \mathrm{~dB}$ over the case for the single fiber analyzed in the configuration factor study, and will mount to the drop tube through a $23 / 4$ inch flange. The bandpass filter center wavelengths will be chosen depending on the estimated initial temperature of the sample (different materials are typically heated to different initial temperatures in the induction furnace). Barium fluoride infrared lenses will be used to focus the outputs onto two cryogenically cooled indium antimonide detectors. Following amplification of the detector outputs, the ratio of the two signals are taken and the temperature derived. 


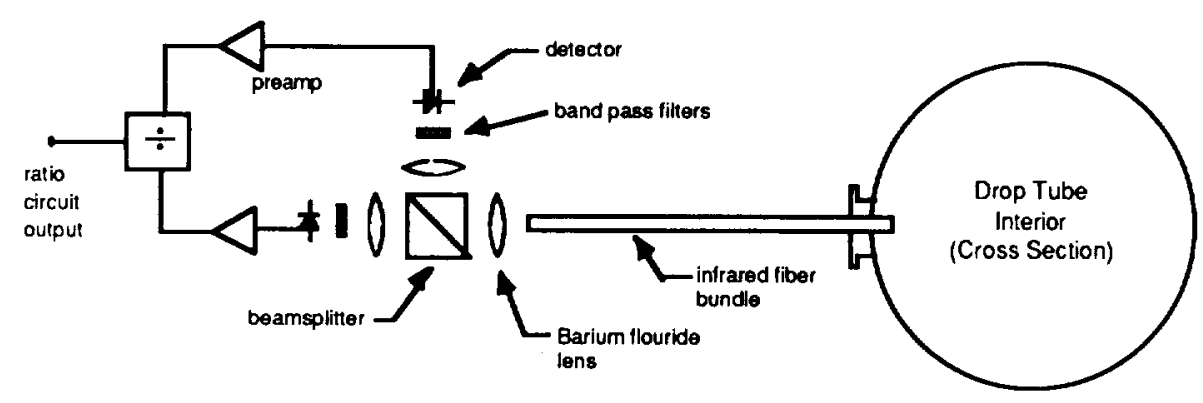

Figure 4. Pyrometer design

\section{CONCLUSIONS}

The work reported focused on determining the feasibility of employing an optical pyrometry approach using infrared optical fibers to measure the temperature of falling particles in the NASA Marshall Space Flight Center drop tube. A mathematical model based on radiative transfer principles was derived, and key parameter values representative of the drop tube system, such as particle size, tube diameter and length, and particle temperature, were used to determine an estimate of the radiant flux that will be incident on the face of an optical fiber used to collect radiation from the incandescent falling particle. The results indicate that the total power collected will be on the order of picowatts. An extension of this work examined the advantage of inclining or tilting the collecting fiber to increase the time that the falling particle remains in the fiber field-of-view. Those results indicate that increases in total power collected of about $15 \%$ may be realized by tilting the fiber. Current work is proceeding towards implementing an experimental pyrometer utilizing the design principles discussed.

\section{REFERENCES}

1. R.O. Claus and J. Cantrell, Proc. IEEE Region 3 Conference, April 1980.

2. R. Vanzetti, "The role of fiber optics in remote temperature measurement," NASA Noncontact Temperature Mcasurement Workshop, April 30, 1987, Washington, DC.

3. K.A. Wickersheim and M.H. Sun, "Fiberoptic thermometry and its application," J. Microwave Powcr, 1987, p 85-93.

4. P.B. Coates, "Multiwavelength pyrometry," Metrologia 17, p. 103, 1981. 